\title{
ON $p$-BIHARMONIC SUBMANIFOLDS IN NONPOSITIVELY CURVED MANIFOLDS
}

\author{
Xiangzhi CaO and Yong Luo
}

\begin{abstract}
Let $u:(M, g) \rightarrow(N, h)$ be a map between Riemannian manifolds $(M, g)$ and $(N, h)$. The $p$-bienergy of $u$ is $\tau_{p}(u)=\int_{M}|\tau(u)|^{p} d v_{g}$, where $\tau(u)$ is the tension field of $u$ and $p>1$. Critical points of $\tau_{p}$ are called $p$-biharmonic maps and isometric $p$-biharmonic maps are called $p$-biharmonic submanifolds. When $p=2, p$-biharmonic submanifolds are biharmonic submanifolds and in recent years many nonexistence results are found for biharmonic submanifolds in nonpositively curved manifolds. In this paper we will study the nonexistence result for general $p$-biharmonic submanifolds.
\end{abstract}

\section{Introduction}

In the past several decades harmonic map plays a central role in geometry and analysis. Let $u:\left(M^{m}, g\right) \rightarrow\left(N^{n}, h\right)$ be a map between Riemannian manifolds $(M, g)$ and $(N, h)$. The energy of $u$ is defined by

$$
E(u)=\int_{M} \frac{|d u|^{2}}{2} d v_{g}
$$

where $d v_{g}$ is the volume element on $(M, g)$.

The Euler-Lagrange equation of $E$ is

$$
\tau(u)=\sum_{i=1}^{m}\left\{\tilde{\nabla}_{e_{i}} d u\left(e_{i}\right)-d u\left(\nabla_{e_{i}} e_{i}\right)\right\}=0,
$$

where $\tilde{\nabla}$ is the Levi-Civita connection on the pullback bundle $u^{-1} T N$ and $\left\{e_{i}\right\}$ is a local orthonormal frame field on $M$. In 1983, Eells and Lemaire [11] proposed to consider the bienergy functional

$$
E_{2}(u)=\int_{M} \frac{|\tau(u)|^{2}}{2} d v_{g}
$$

2010 Mathematics Subject Classification. Primary 53B25; Secondary 53C42.

Key words and phrases. p-biharmonic submanifolds, Chen's conjecture, Liouville type theorem. Received December 2, 2015; revised February 26, 2016. 
where $\tau(u)$ is the tension field of $u$. Recall that $u$ is harmonic if $\tau(u)=0$. The Euler-Lagrange equation for $E_{2}$ is

$$
\tau_{2}(u):=\tilde{\triangle} \tau(u)-\sum_{i=1}^{m} R^{N}\left(\tau(u), d u\left(e_{i}\right)\right) d u\left(e_{i}\right)=0 .
$$

To further generalize the notion of harmonic maps, Hornung and Moser (see also [14]) considered the $p$-bienergy $(p>1)$ functional as follows:

$$
E_{p}(u)=\int_{M}|\tau(u)|^{p} d v_{g}
$$

The $p$-bitension field $\tau_{p}(u)$ is

$$
\tau_{p}(u):=\tilde{\triangle}\left(|\tau(u)|^{p-2} \tau(u)\right)-\sum_{i=1}^{m}\left(R^{N}\left(|\tau(u)|^{p-2} \tau(u), d u\left(e_{i}\right)\right) d u\left(e_{i}\right)\right) .
$$

The Euler-Lagrange equation for $E_{p}$ is $\tau_{p}(u)=0$ and a map $u$ satisfying $\tau_{p}(u)=0$ is called $p$-biharmonic maps. If furthermore $u:\left(M^{m}, g\right) \rightarrow\left(N^{n}, h\right)$ is an isometry immersion, then we call $u$ p-biharmonic submanifold and 2-biharmonic submanifolds are called biharmonic submanifolds.

For biharmonic submanifolds, we have the well known Chen's conjecture [6]:

Chen's conjecture: Every biharmonic submanifold in $\mathbf{E}^{n}$ is minimal.

Chen's conjecture inspires the research on the nonexistence of biharmonic submanifolds in nonpositively curved manifolds ([1]-[10], [12] [17] [18], [20]-[28] etc.). Motivated by Chen's conjecture, Han [13] proposed the following conjecture:

Conjecture: Every complete $p$-biharmonic submanifolds in non-positively curved Riemannian manifold is minimal.

In [13], using the method developed in [21], Han proved several results on the nonexistence of $p$-biharmonic submanifolds.

Remark 1.1. In Han's paper, he defined p-biharmonic submanifolds to be isometric $p$-biharmonic maps where he required $p \geq 2$, but that is not necessary. His nonexistence results also hold for $p$-biharmonic submanifolds $(p>1)$, without any change in the proof.

In this paper, using the method in [21] again, we get:

THeOREM 1.2. Suppose $(M, g)$ is a complete p-biharmonic submanifold in $(N, h)$ with non-positive sectional curcvature (Ricci curvature if $M$ is a hypersurface). If $M$ is of at most polynomial volume growth, it is minimal.

Here we say $(M, g)$ is of at most polynomial volume growth, if for a fixed point $x_{0} \in M$ and geodesic ball $B_{r}\left(x_{0}\right), \operatorname{Vol}\left(B_{r}\left(x_{0}\right)\right) \leq C(1+r)^{s}$ for some positive integer $s$ and $C$ independent of $r$. 
For $p$-biharmonic hypersurfaces in a Euclidean space, we have

THEOREM 1.3. Every weakly convex p-biharmonic hypersurface in a Euclidean space is minimal.

We say a hypersurface $M$ in $N$ is weakly convex, if its principle curvatures are nonnegative. When $p=2$, this result was proved in [20]. Furthermore we have

THEOREM 1.4. Every weakly convex p-biharmonic hypersurface in a nonpositive curvature space form is minimal.

Remark 1.5. After our paper is completed, the second named author was informed by Yingbo Han that theorem 1.4 was also proved in [15].

We also have

THEOREM 1.6. Every p-biharmonic submanifold properly immersed in a Euclidean space is minimal.

We say a map is proper if the preimage of any compact subset is compact. When $p=2$, this theorem was proved in [1].

The rest of this paper is organized as follows: In section 2 we will briefly recall the theory of $p$-biharmonic submanifolds and submanifold theory. Our main theorems are proved in section 3 .

\section{Preliminaries}

In this section we give more details on the definitions of harmonic maps, biharmonic maps, $p$-biharmonic maps and $p$-biharmonic submanifolds.

Let $u:\left(M^{m}, g\right) \rightarrow\left(N^{n}, h\right)$ be a map from an $m$-dimensional Riemannian manifold $(M, g)$ to an $n$-dimensional Riemannian manifold $(N, h)$. The energy of $u$ is defined by

$$
E(u)=\int_{M} \frac{|d u|^{2}}{2} d v_{g} .
$$

The Euler-Lagrange equation of $E$ is

$$
\tau(u):=\sum_{i=1}^{m}\left\{\tilde{\nabla}_{e_{i}} d u\left(e_{i}\right)-d u\left(\nabla_{e_{i}} e_{i}\right)\right\}=0,
$$

where we denote $\nabla$ the Levi-Civita connection on $(M, g)$, and $\tilde{\nabla}$ the induced LeviCivita connection of the pullback bundle $u^{-1} T N$. A map $u:\left(M^{m}, g\right) \rightarrow\left(N^{n}, h\right)$ 
is called a harmonic map if $\tau(u)=0$. To generalize the notion of harmonic maps, Eells and Lemaire [11] proposed to consider the bienergy functional

$$
E_{2}(u)=\int_{M} \frac{|\tau(u)|^{2}}{2} d v_{g}
$$

The Euler-Lagrange equation for $E_{2}$ is (see [19])

$$
\tau_{2}(u):=\tilde{\triangle} \tau(u)-\sum_{i=1}^{m} R^{N}\left(\tau(u), d u\left(e_{i}\right)\right) d u\left(e_{i}\right)=0 .
$$

To further generalize the notion of harmoic maps, Han and Feng [14] (see also [16]) introduced the $F$-bienergy functional

$$
E_{F}(u)=\int_{M} F\left(\frac{|\tau(u)|^{2}}{2}\right) d v_{g}
$$

where $F:[0,+\infty)$ and $F^{\prime}(x)>0$ if $x>0$.

The critical points of the $F$-bienergy functional with $F(x)=(2 x)^{p / 2}(p>1)$ are called $p$-biharmonic maps and isometric $p$-biharmonic maps are called $p$-biharmonic submanifolds.

The $p$-bitension field $\tau_{p}(u)$ is

$$
\tau_{p}(u):=\tilde{\triangle}\left(|\tau(u)|^{p-2} \tau(u)\right)-\sum_{i=1}^{m}\left(R^{N}\left(|\tau(u)|^{p-2} \tau(u), d u\left(e_{i}\right)\right) d u\left(e_{i}\right)\right) .
$$

A $p$-biharmonic map satisfies $\tau_{p}(u)=0$.

Now we briefly recall the submanifold theory. Let $u:(M, g) \rightarrow(N, h)$ be an isometric immersion from an $m$-dimensional Riemannian manifold into an $m+t(t \geq 1)$-dimensional Riemannian manifold. The second fundamental form $B: T M \times T M \rightarrow T^{\perp}(M)$ is defined by:

$$
\bar{\nabla}_{X} Y=\nabla_{X} Y+B(X, Y), \quad X, Y \in \Gamma(T M),
$$

where $\bar{\nabla}$ is the Levi-Civita connection on $N$ and $\nabla$ is the Levi-Civita connection on $M$. The Weingarten formula is given by

$$
\bar{\nabla}_{X} \xi=-A_{\xi} X+\nabla_{X}^{\perp} \xi, \quad X \in \Gamma(T M),
$$

where $A_{\xi}$ is called the Weingarten map w.r.t. $\xi \in T^{\perp} M$, and $\nabla^{\perp}$ denotes the normal connection on the normal bundle of $M$ in $N$. For any $x \in M$, the mean curvature vector field $H$ of $M$ at $x$ is

$$
\vec{H}=\frac{1}{m} \sum_{i=1}^{m} B\left(e_{i}, e_{i}\right)
$$


If $u$ is an isometric immersion, we see that $\left\{d u\left(e_{i}\right)\right\}$ is a local orthonormal frame of $M$. In addition, for any $X, Y \in \Gamma(T M)$,

$$
\nabla d u(X, Y)=\tilde{\nabla}_{X}(d u(Y))-d u\left(\nabla_{X}^{Y}\right)=B(X, Y),
$$

where $\tilde{\nabla}$ is the connection on the pull back bundle $u^{-1} T N$, whose fiber at a point $x \in M$ is $T_{u(x)} N=T^{\perp} M \oplus T M$. Therefore if $u$ is an isometric immersion,

$$
\tau(u)=\operatorname{tr} \nabla d u=\operatorname{tr} B=m \vec{H},
$$

and a $p$-biharmonic submanifold satisfies the following equatuion:

$$
\tau_{p}(u)=\tilde{\triangle}\left(|\vec{H}|^{p-2} \vec{H}\right)-\sum_{i=1}^{m}\left(R^{N}\left(|\vec{H}|^{p-2} \vec{H}, e_{i}\right) e_{i}\right)=0,
$$

where $\tilde{\triangle}=\sum_{i=1}^{m}\left(\tilde{\nabla}_{e_{i}} \tilde{\nabla}_{e_{i}}-\tilde{\nabla}_{\nabla_{e_{i} e_{i}}}\right), \tilde{\nabla}$ is the connection on the pullback bundle, and $R^{N}$ is the Riemanian curvature tensor on $N$.

From (2.3), we get for any vector field $\xi \in \Gamma\left(T^{\perp} M\right)$ :

$$
\begin{aligned}
\tilde{\nabla}_{e_{i}} \tilde{\nabla}_{e_{i}} \xi & =\tilde{\nabla}_{e_{i}}\left(\nabla_{e_{i}}^{\perp} \xi-A_{\xi} e_{i}\right) \\
& =\nabla_{e_{i}}^{\perp} \nabla_{e_{i}}^{\perp} \xi-\tilde{\nabla}_{e_{i}} A_{\xi} e_{i}-A_{\nabla_{e_{i}}^{\perp} \xi} e_{i} \\
& =\nabla_{e_{i}}^{\perp} \nabla_{e_{i}}^{\perp} \xi-\nabla_{e_{i}} A_{\xi} e_{i}-B\left(e_{i}, A_{\xi} e_{i}\right)-A_{\nabla_{e_{i}}^{\perp} \xi}\left(e_{i}\right),
\end{aligned}
$$

and

$$
\tilde{\nabla}_{\nabla_{e_{i}} e_{i}} \xi=\nabla_{\nabla_{e_{i}} e_{i}}^{\perp} \xi-A_{\xi}\left(\nabla_{e_{i}} e_{i}\right) .
$$

Combining the above two identities, we get

$$
\begin{aligned}
\tilde{\triangle} \xi & =\nabla_{e_{i}}^{\perp} \nabla_{e_{i}}^{\perp} \xi-\nabla_{e_{i}} A_{\xi} e_{i}-B\left(e_{i}, A_{\xi} e_{i}\right)-A_{\nabla_{e_{i}}^{\perp}}\left(e_{i}\right)-\nabla_{\nabla_{e_{i}} e_{i}}^{\perp} \xi+A_{\xi}\left(\nabla_{e_{i}} e_{i}\right) \\
& =\triangle^{\perp} \xi-\left(\nabla_{e_{i}} A_{\xi}\right) e_{i}+A_{\xi}\left(\nabla_{e_{i}} e_{i}\right)-B\left(e_{i}, A_{\xi} e_{i}\right)-A_{\nabla_{e_{i}}^{\perp}}\left(e_{i}\right) .
\end{aligned}
$$

Therefore by decomposing the $p$-biharmonic submanifold equation into its normal and tangential parts respectively we get ([13]):

$$
\begin{aligned}
& \Delta^{\perp}\left(|\vec{H}|^{p-2} \vec{H}\right)-\sum_{i=1}^{m} B\left(A_{|\vec{H}|^{p-2} \vec{H}} e_{i}, e_{i}\right)+\sum_{i=1}^{m}\left(R^{N}\left(|\vec{H}|^{p-2} \vec{H}, e_{i}\right) e_{i}\right)^{\perp}=0 \\
& \operatorname{Tr}_{g}\left(\nabla A_{|\vec{H}|^{p-2} \vec{H}}\right)+\operatorname{Tr}_{g}\left[A_{\nabla^{\perp}|\vec{H}|^{p-2} \vec{H}}(\cdot)\right]-\sum_{1}^{m}\left(R^{N}\left(|\vec{H}|^{p-2} \vec{H}, e_{i}\right) e_{i}\right)^{\top}=0
\end{aligned}
$$




\section{Proof of theorems}

\subsection{Proof of Theorem 1.2}

Proof. From equation (2.6), we see that

$$
\begin{aligned}
\Delta|\vec{H}|^{2 p-2}= & \left.2\left|\nabla\left(|\vec{H}|^{p-2} \vec{H}\right)\right|^{2}+2\left\langle\triangle\left(|\vec{H}|^{p-2} \vec{H}\right),|\vec{H}|^{p-2} \vec{H}\right)\right\rangle \\
= & 2\left|\nabla\left(|\vec{H}|^{p-2} \vec{H}\right)\right|^{2}+2\left\langle\sum_{i=1}^{m} B\left(A_{|\vec{H}|^{p-2} \vec{H}} e_{i}, e_{i}\right),|\vec{H}|^{p-2} \vec{H}\right\rangle \\
& -2\left\langle R^{N}\left(e_{i},|\vec{H}|^{p-2} \vec{H}\right) e_{i},|\vec{H}|^{p-2} \vec{H}\right\rangle \\
\geq & \left.2\left|\nabla\left(|\vec{H}|^{p-2} \vec{H}\right)\right|^{2}+2\left\langle\sum_{i=1}^{m} B\left(A_{|\vec{H}|^{p-2} \vec{H}} e_{i}, e_{i}\right),|\vec{H}|^{p-2}\right) \vec{H}\right\rangle,
\end{aligned}
$$

where we used (2.6) and the fact that the sectional curvature of $N$ is nonpositive. Therefore by the following inequality ([13])

$$
\sum_{i=1}^{m}\left\langle B\left(A_{|\vec{H}|^{p-2} \vec{H}} e_{i}, e_{i}\right),|\vec{H}|^{p-2} \vec{H}\right\rangle \geq m|\vec{H}|^{2 p},
$$

we obtain

$$
\Delta|\vec{H}|^{2 p-2} \geq 2|\vec{H}|^{2 p-4}|\nabla \vec{H}|^{2}+2 m|\vec{H}|^{2 p} .
$$

For a fixed point $x_{0} \in M$, and every $r>0$, let us consider the following cut off function $\lambda(x)$ on $M$ :

$$
\begin{cases}0 \leq \lambda(x) \leq 1 & x \in M \\ \lambda(x)=1 & x \in B_{r}\left(x_{0}\right) \\ \lambda(x)=0 & x \in M \backslash B_{2 r}\left(x_{0}\right) \\ |\nabla \lambda(x)| \leq \frac{C}{r} & x \in M\end{cases}
$$

where $C$ is independent of $r$, and $B_{r}\left(x_{0}\right)$ is a geodesic ball of $M$.

Let $a \geq 2$, and $b$ be positive constants to be determined later. From (3.3), we have

$$
\begin{aligned}
-\int_{M} \nabla\left(\lambda^{b}|\vec{H}|^{a}\right) \cdot \nabla\left(|\vec{H}|^{2 p-2}\right) & =\int_{M} \lambda^{b}|\vec{H}|^{a} \triangle\left(|\vec{H}|^{2 p-2}\right) \\
& \geq \int_{M} 2 \lambda^{b}|\vec{H}|^{a}\left(|\vec{H}|^{2 p-4}|\nabla \vec{H}|^{2}+m|\vec{H}|^{2 p}\right) \\
& \geq \int_{M} 2 \lambda^{b}|\vec{H}|^{a+2 p-4}|\nabla \vec{H}|^{2}+2 m \int_{M} \lambda^{b}|\vec{H}|^{a+2 p},
\end{aligned}
$$


where $\nabla f \cdot \nabla g:=g^{i j} \frac{\partial f}{\partial x_{i}} \frac{\partial g}{\partial x_{j}}$ in local coordinates, for $f, g \in C^{1}(M)$, and for simplicity we omit the symbol · in the subsequent.

On the other hand, we have

$$
\begin{aligned}
-\int_{M} \nabla\left(\lambda^{b}|\vec{H}|^{a}\right) \nabla|\vec{H}|^{2 p-2}= & -\int_{M}\left(b \lambda^{b-1} \nabla \lambda|\vec{H}|^{a}+\lambda^{b} a|\vec{H}|^{a-2}\langle\vec{H}, \nabla \vec{H}\rangle\right) \\
& \times\left((2 p-2)|\vec{H}|^{2 p-4}\langle\vec{H}, \nabla \vec{H}\rangle\right) \\
= & -\int_{M}(2 p-2) b \lambda^{b-1} \nabla \lambda|\vec{H}|^{a+2 p-4}\langle\vec{H}, \nabla \vec{H}\rangle \\
& -(2 p-2) a \int_{M} \lambda^{b}|\vec{H}|^{a+2 p-6}\langle\vec{H}, \nabla \vec{H}\rangle^{2} \\
\leq & -\int_{M}(2 p-2) b \lambda^{b-1} \nabla \lambda|\vec{H}|^{a+2 p-4}\langle\vec{H}, \nabla \vec{H}\rangle .
\end{aligned}
$$

Let $b=a+2 p$, we have

$$
\begin{aligned}
2 \int_{M} & \lambda^{a+2 p}|\vec{H}|^{a+2 p-4}|\nabla \vec{H}|^{2}+2 m \int_{M} \lambda^{a+2 p}|\vec{H}|^{a+2 p} \\
& \leq-(2 p-2)(a+2 p) \int_{M} \lambda^{a+2 p-1} \nabla \lambda|\vec{H}|^{a+2 p-4}\langle\vec{H}, \nabla \vec{H}\rangle \\
& \leq \int_{M} \lambda^{a+2 p}|\vec{H}|^{a+2 p-4}|\nabla \vec{H}|^{2}+\int_{M} \lambda^{a+2 p}|\vec{H}|^{a+2 p}+C(a, p) \int_{M}|\nabla \lambda|^{a+2 p}
\end{aligned}
$$

where in the last inequality we used the following young's inequality twice:

$$
a b \leq \varepsilon^{p} \frac{a^{p}}{p}+\frac{1}{\varepsilon^{q}} \frac{b^{q}}{q}
$$

where $\epsilon>0$ and $p, q$ are positive constants satisfying $\frac{1}{p}+\frac{1}{q}=1$.

From (3.7), we see that if $M$ is of at most polynomial volume growth then

$$
(2 m-1) \int_{B_{r}\left(x_{0}\right)}|\vec{H}|^{a+2 p} d v_{g} \leq C(a, p) \frac{1}{r^{a+2 p}}(1+2 r)^{s} .
$$

We finish the proof by letting $a$ be big enough and $r \rightarrow+\infty$.

\subsection{Proof of Theorem 1.3 and Theorem 1.4}

\subsubsection{Proof of Theorem 1.3}

Proof. From (2.6) and (2.7) we have:

$$
\begin{gathered}
\Delta^{\perp}\left(|\vec{H}|^{p-2} \vec{H}\right)-\sum_{i=1}^{m} B\left(A_{|\vec{H}|^{p-2} \vec{H}} e_{i}, e_{i}\right)=0, \\
\operatorname{Tr}_{g}\left(\nabla A_{|\vec{H}|^{p-2} \vec{H}}\right)+\operatorname{Tr}_{g}\left[A_{\nabla^{\perp}|\vec{H}|^{p-2} \vec{H}}(\cdot)\right]=0 .
\end{gathered}
$$


Note that for any $X, Y \in T M$ and $\xi \in T^{\perp} M$ we have

$$
\left\langle A_{\xi} X, Y\right\rangle=\langle B(X, Y), \xi\rangle \text {. }
$$

Assume that $\vec{H}=H v$, where $v$ is the unit normal vector field on $M$. Note that by the assumption that $M$ is weakly convex, we have $H \geq 0$. Define

$$
B=\{p \in M: H(p)>0\} .
$$

We will prove that $B$ is an empty set by a contradiction argument, and so $M$ is minimal and we are done. If $B$ is not empty, we see that $B$ is an open subset of $M$. We assume that $B_{1}$ is a nonempty connect component of $B$. We will prove that $H=0$ in $B_{1}$, thus a contradiction. We prove it in two steps.

STEP 1. $H$ is a constant in $B_{1}$.

Let $q \in B_{1}$ be a point. Around $q$ we choose a local orthonormal frame $\left\{e_{k}, k=1, \ldots, m\right\}$ such that $\nabla_{e_{i}} e_{j}(q)=0$ and $\langle h, v\rangle$ is a diagonal matrix at $q$, where $v$ is the unit normal vector field of $M$. For any $1 \leq k \leq m$, we have at $q$

$$
\begin{aligned}
\left\langle\sum_{i=1}^{m} A_{\nabla_{e_{i}}^{\perp}|\vec{H}|^{p-2} \vec{H}} e_{i}, e_{k}\right\rangle & =\sum_{i=1}^{m}\left\langle B\left(e_{i}, e_{k}\right), \nabla_{e_{i}}^{\perp}|\vec{H}|^{p-2} \vec{H}\right\rangle \\
& =\left\langle B\left(e_{k}, e_{k}\right), \nabla_{e_{k}}^{\perp}|\vec{H}|^{p-2} \vec{H}\right\rangle
\end{aligned}
$$

From (3.10) and (3.11), we have at $q$

$$
\begin{aligned}
0 & =\sum_{i=1}^{m}\left\langle\left(\nabla_{e_{i}} A_{|\vec{H}|^{p-2} \vec{H}}\right) e_{i}, e_{k}\right\rangle+\left\langle\sum_{i=1}^{m} A_{\nabla_{e_{i}}^{\perp}|\vec{H}|^{p-2} \vec{H}} e_{i}, e_{k}\right\rangle \\
& =\sum_{i=1}^{m} e_{i}\left\langle A_{|\vec{H}|^{p-2} \vec{H}} e_{i}, e_{k}\right\rangle+\left\langle\sum_{i=1}^{m} A_{\nabla_{e_{i}}^{\perp}|\vec{H}|^{p-2} \vec{H}} e_{i}, e_{k}\right\rangle \\
& =\sum_{i=1}^{m} e_{i}\left\langle B\left(e_{i}, e_{k}\right),|\vec{H}|^{p-2} \vec{H}\right\rangle+\left\langle\sum_{i=1}^{m} A_{\nabla_{e_{i}}^{\perp}|\vec{H}|^{p-2} \vec{H}} e_{i}, e_{k}\right\rangle \\
& =\sum_{i=1}^{m}\left\langle|\vec{H}|^{p-2} \vec{H}, \nabla_{e_{i}}^{\perp} B\left(e_{i}, e_{k}\right)\right\rangle+2\left\langle\sum_{i=1}^{m} A_{\nabla_{e_{i}}^{\perp}|\vec{H}|^{p-2} \vec{H}} e_{i}, e_{k}\right\rangle \\
& =\sum_{i=1}^{m}\left\langle|\vec{H}|^{p-2} \vec{H}, \nabla_{e_{k}}^{\perp} B\left(e_{i}, e_{i}\right)\right\rangle+2\left\langle\sum_{i=1}^{m} A_{\nabla_{e_{i}}^{\perp}|\vec{H}|^{p-2} \vec{H}} e_{i}, e_{k}\right\rangle \\
& =m|\vec{H}|^{p-1} \nabla_{e_{k}} H+2\left\langle B\left(e_{k}, e_{k}\right), \nabla_{e_{k}}^{\perp}|\vec{H}|^{p-2} \vec{H}\right\rangle \\
& =m|\vec{H}|^{p-1} \nabla_{e_{k}} H+2 \lambda_{k}\left\langle v, \nabla_{e_{k}}^{\perp}|\vec{H}|^{p-2} \vec{H}\right\rangle
\end{aligned}
$$




$$
\begin{aligned}
& =m|\vec{H}|^{p-1} \nabla_{e_{k}} H+2 \lambda_{k}\left\langle v,(p-2)|\vec{H}|^{p-2} \nabla_{e_{k}} \vec{H}+|\vec{H}|^{p-2} \nabla_{e_{k}} \vec{H}\right\rangle \\
& =m|\vec{H}|^{p-1} \nabla_{e_{k}} H+2 \lambda_{k}(p-1)|\vec{H}|^{p-2}\left\langle v, \nabla_{e_{k}} \vec{H}\right\rangle \\
& =\left(m|\vec{H}|^{p-1}+2 \lambda_{k}(p-1)|\vec{H}|^{p-2}\right) \nabla_{e_{k}} H,
\end{aligned}
$$

where in the fifth equality we used the Codazzi equation.

Because $\lambda_{k} \geq 0, p>1$, we have $\left(m|\vec{H}|^{p-1}+2 \lambda_{k}(p-1)|\vec{H}|^{p-2}\right)>0$ and so

$$
\nabla_{e_{k}} H(q)=0,
$$

for any $k=1, \ldots, m$, which implies that

$$
\nabla H(q)=0 .
$$

Because $q$ is an arbitrary point in $B_{1}$, we see that in $B_{1}$

$$
\nabla H=0 .
$$

Therefore we get that $H$ is a constant in $B_{1}$ and $\triangle|\vec{H}|^{2 p-2}=0$.

STEP 2. $H$ is zero in $B_{1}$.

Let $q \in B_{1}$, by step 1 , we see that $\triangle|\vec{H}|^{2 p-2}=0$. On the other hand, by equation (3.9), we have

$$
\begin{aligned}
\triangle|\vec{H}|^{2 p-2} & =2\left|\nabla\left(|\vec{H}|^{p-2}\right) \vec{H}\right|^{2}+2\left\langle|\vec{H}|^{p-2} \vec{H}, \triangle\left(|\vec{H}|^{p-2} \vec{H}\right)\right\rangle \\
& \geq 2\left\langle B\left(A_{|\vec{H}|^{p-2} \vec{H}}, e_{i}\right),|\vec{H}|^{p-2} \vec{H}\right\rangle \\
& =2\left\langle A_{|\vec{H}|^{p-2} \vec{H}} e_{i}, A_{|\vec{H}|^{p-2} \vec{H}} e_{i}\right\rangle \\
& =2|\vec{H}|^{2 p-2}\left\langle A_{v} e_{i}, A_{v} e_{i}\right\rangle \\
& \geq 2 m|\vec{H}|^{2 p}
\end{aligned}
$$

Therefore $H=0$ in $B_{1}$, a contradiction. This completes the proof of Theorem 1.3 .

\subsubsection{Proof of Theorem 1.4}

Proof. The proof of Theorem 1.4 is similar with the proof of Theorem 1.3. If $M$ is a $p$-biharmonic submanifold in the space form $N(c)(c \leq 0)$, then it satisfies the following two equations:

$$
\begin{gathered}
\Delta^{\perp}\left(|\vec{H}|^{p-2} \vec{H}\right)-\sum_{i=1}^{m} B\left(A_{|\vec{H}|^{p-2} \vec{H}} e_{i}, e_{i}\right)+c|\vec{H}|^{p-2} \vec{H}=0 \\
\operatorname{Tr}_{g}\left(\nabla A_{|\vec{H}|^{p-2} \vec{H}}\right)+\operatorname{Tr}_{g}\left[A_{\nabla^{\perp}|\vec{H}|^{p-2} \vec{H}}(\cdot)\right]=0 .
\end{gathered}
$$


From equation (3.18) we will get that if $H$ is not identically zero in a nonempty component, then it must be a constant and by equation (3.17) we can deduce that it is zero, a contradiction.

\subsection{Proof of Theorem 1.6}

Proof. We will prove the theorem by a contradiction argument. Suppose that $\vec{H}\left(x_{0}\right) \neq 0$ for some $x_{0} \in M$.

Set $u(x)=|\vec{H}(x)|^{2 p-2}$ for $x \in M$. For each $\rho>0$ let

$$
F(x)=F_{\rho}(x)=\left(\rho^{2}-|X(x)|^{2}\right)^{2 p-2} u(x),
$$

for $x \in M \cap X^{-1}\left(\bar{B}_{\rho}\right)$, where $X: M \rightarrow \mathbf{R}^{n}$ is the isometric immersion and $B_{\rho}$ is the standard ball in $\mathbf{R}^{n}$ with radius $\rho$.

Assume that $x_{0} \in X^{-1}\left(B_{\rho_{0}}\right)$. For each $\rho \geq \rho_{0}, F=F_{\rho}$ is a nonnegative function which is not identically zero on $M \cap X^{-1}\left(\bar{B}_{\rho}\right)$. Assume that $q \in M \cap$ $X^{-1}\left(B_{\rho}\right)$ is the maximum point of $F$. Then $\nabla F(q)=0$ and hence we get at $q$

$$
\frac{\nabla u}{u}=\frac{(2 p-2) \nabla|X(x)|^{2}}{\rho^{2}-|X(x)|^{2}}
$$

By $\Delta F(q) \leq 0$ we get at $q$

$$
\frac{\Delta u}{u} \leq \frac{\left.\left.(2 p-2)(2 p-1)|\nabla| X(q)\right|^{2}\right|^{2}}{\left(\rho^{2}-|X(q)|^{2}\right)^{2}}+\frac{(2 p-2) \Delta|X(q)|^{2}}{\rho^{2}-|X(q)|^{2}} .
$$

By $\Delta X=m \vec{H}$ we see that

$$
\left.\left.|\nabla| X(x)\right|^{2}\right|_{g} ^{2} \leq 4 m|X(x)|^{2}
$$

and

$$
\Delta|X(x)|^{2}=2 \sum_{i=1}^{m}\left|\nabla_{e_{i}} X(x)\right|^{2}+2\langle X(x), \Delta X(x)\rangle \leq 2 m+2 m|X(x)||\vec{H}(x)|,
$$

where $m=\operatorname{dim} M . \quad$ Recall that $\Delta|\vec{H}|^{2 p-2} \geq 2 m|\vec{H}|^{2 p}$, i.e. $\Delta u \geq u^{2 p /(2 p-2)}$, thus we obtain

$$
u(q)^{1 /(p-1)} \leq \frac{2(2 p-2)(2 p-1)|X(q)|^{2}}{\left(\rho^{2}-|X(q)|^{2}\right)^{2}}+\frac{(2 p-2)\left(1+|X(q)| u^{1 /(2 p-2)}\right)}{\rho^{2}-|X(q)|^{2}} .
$$

From the last inequality one gets

$$
\begin{aligned}
u(q) \leq C(p)[ & \frac{|X(q)|^{2 p-2}}{\left(\rho^{2}-|X(q)|^{2}\right)^{2 p-2}}+\frac{1}{\left(\rho^{2}-|X(q)|^{2}\right)^{p-1}} \\
& \left.+\sqrt{u(q)}|X(q)|^{p-1} \frac{1}{\left(\rho^{2}-|X(q)|^{2}\right)^{p-1}}\right]
\end{aligned}
$$


where $C(p)$ is a constant depends only on $p$. Therefore

$$
F(q) \leq C(p)\left[|X(q)|^{2 p-2}+\left(\rho^{2}-|X(q)|^{2}\right)^{p-1}+\sqrt{F(q)}|X(q)|^{p-1}\right],
$$

which implies that

$$
F(q) \leq C(p) \rho^{2 p-2} .
$$

Since $q$ is the maximum of $F$, for any $x \in M \cap B_{\rho}$ we have

$$
F(x) \leq F(q) \leq C(p) \rho^{2 p-2} .
$$

Therefore

$$
|\vec{H}(x)|^{2 p-2} \leq \frac{C(p) \rho^{2 p-2}}{\left(\rho^{2}-|X(x)|^{2}\right)^{2 p-2}},
$$

for any $x \in M \cap B_{\rho}$ and $\rho \geq \rho_{0}$. Letting $\rho \rightarrow+\infty$ in the last inequality and $x=x_{0}$ we get $\left|\vec{H}\left(x_{0}\right)\right|=0$, a contradiction. Therefore $M$ is minimal.

Acknowledgement. The second named author would like to thank Professor Yingbo Han for stimulating discussions and he is partially supported by the Postdoctoral Science Foundation of China (No. 2015M570660), and the Projectsponsored by SRF for ROCS, SEM. The authors are appreciated with the referee for his or her suggestions and comments which made this paper more readable.

\section{REFERENCES}

[1] K. Akutagawa and S. Maeta, Biharmonic properly immersed submanifolds in Euclidean spaces, Geom. Dedicata 164 (2013), 351-355.

[2] A. Balmus, S. Montaldo and C. Oniciuc, Biharmonic hypersurfaces in 4-dimensional space forms, Math. Nachr. 283 (2010), 1696-1705.

[ 3 ] R. Caddeo, S. Montaldo and C. Oniciuc, Biharmonic submanifolds of $\mathbf{S}^{3}$, Int. J. Math. 12 (2001), 867-876.

[4] R. Caddeo, S. Montaldo and C. Oniciuc, On biharmonic maps, Contemp. Math. 288 (2001), 286-290.

[ 5 ] R. Caddeo, S. Montaldo AND C. Oniciuc, Biharmonic submanifolds in spheres, Israel J. Math. 130 (2002), 109-123.

[6] B. Y. CHEN, Some open problems and conjectures on submanifolds of finite type, Soochow J. Math. 17 (1991), 169-188.

[ 7 ] B. Y. Chen, A report on submanifolds of finite type, Soochow J. Math. 22 (1996), 117-137.

[8] B. Y. Chen AND M. I. Munteanu, Biharmonic ideal hypersurfaces in Euclidean spaces, Differential Geom. Appl. 31 (2013), 1-16.

[9] F. Defever, Hypersurfaces of $E^{4}$ with harmonic mean curvature vector, Math. Nachr. 196 (1998), 61-69.

[10] I. Dimitric, Submanifolds of $E^{m}$ with harmonic mean curvature vector, Bull. Inst. Math. Acad. Sinica 20 (1992), 53-65.

[11] J. Eells and L. Lemaire, Selected topics in harmonic maps, Amer. Math. Soc., CBMS, 50 (1983). 
[12] Y. Fu, Biharmonic hypersurfaces with three distinct principal curvatures in Euclidean 5-space, J. Geom. Phy. 75 (2014), 113-119.

[13] Y. B. HAN, Some results of $p$-biharmonic submanifolds in a Riemannian manifold of nonpositive, J. Geom. 106 (2015), 471-482.

[14] Y. B. Han and S. X. Feng, Some results of $F$-biharmonic maps, Acta Math. Univ. Comenianae 83 (2014), 47-66.

[15] Y. B. Han and W. Zhang, Some results of $p$-biharmonic maps into a non-positively curved manifold, J. Korean Math. Soc. 52 (2015), 1097-1108.

[16] P. Hornung ANd R. Moser, Intrinsically p-biharmonic maps, Cal. Var. and Pdes 51 (2014), 597-620.

[17] T. Hasanis and T. Vlachos, Hypersurfaces in $E^{4}$ with harmonic mean curvature vector field, Math. Nachr. 172 (1995), 145-169.

[18] G. Y. JIANG, 2-harmonic isometric immersions between Riemannian manifolds, Chinese Ann. Math. Ser. A. 7 (1986), 130-144.

[19] G. Y. JIANG, 2-harmonic maps and their first and second variational formulas, Chinese Ann. Math. Ser. A. 7 (1986), 389-402.

[20] Y. Luo, Weakly convex biharmonic hypersurfaces in nonpositive curvature space forms are minimal, Results in Math. 65 (2014), 49-56.

[21] Y. Luo, On biharmonic submanifolds in non-positively curved manifolds, J. Geom. Phy. 88 (2015), 76-87.

[22] S. Matta, Properly immersed submanifolds in complete Riemannian manifolds, Adv. Math. 253 (2014), 139-151.

[23] N. Nakauchi and H. Urakawa, Biharmonic submanifolds in a Riemannian manifold with non-positive curvature, Results. Math. 63 (2013), 467-474.

[24] N. Nakauchi and H. Urakawa, Biharmonic hypersurfaces in a Riemannian manifold with non-positive Ricci curvature, Ann. Glob. Anal. Geom. 40 (2011), 125-131.

[25] N. Nakauchi, H. Urakawa and S. Gudmundsson, Biharmonic maps into a Riemannian manifold of non-positive curvature, Geom. Dedicata 169 (2014), 263-272.

[26] C. OnICIUC, Biharmonic maps between Riemannian manifolds, An. St. Al. Univ. Al. I. Cuza, Iasi, 68 (2002), 237-248.

[27] Y. L. OU, Biharmonic hypersurfaces in Riemannian manifolds, Pacific J. Math. 248 (2010), 217-232.

[28] Y. L. OU AND L. TANG, On the generalized Chen's conjecture on biharmonic submanifolds, Michigan Math. J. 61 (2012), 531-542.

\author{
Xiangzhi Cao \\ School of Mathematics and Statistics \\ WUHAN UNIVERSITY \\ HubeI 430072 \\ P.R. CHINA \\ E-mail: caoxiangzhi@whu.edu.cn \\ Yong Luo \\ SCHOOL OF Mathematics and Statistics \\ WUHAN UNIVERSITY \\ HubeI 430072 \\ P.R. CHINA \\ E-mail: yongluo@whu.edu.cn
}

